\title{
ESTUdo POLAROGRÁFICO SOBRE A DETERMINAÇÃo DE Fe(III) UTILIZANDO-SE A TÉCNICA DA POLAROGRAFIA DE PULSO DIFERENCIAL
}

Luiz Manoel Aleixo, Melissa Sitton e Fabiana Alves de Lima Ribeiro

Departamento de Química Analítica, Instituto de Química, Universidade Estadual de Campinas, CP 6154, 13083-970 Campinas - SP

Recebido em 20/10/00; aceito em 4/3/01

\begin{abstract}
POLAROGRAPHIC STUDY ABOUT THE DETERMINATION OF Fe(III) USING THE TECHNIQUE OF DIFFERENCIAL PULSE POLAROGRAPHY. A differential pulse polarographic study with the objective to determine iron (III) in presense of copper (II) in a supporting electrolyte based on citrate EDTA was made. The best experimental conditions found were a supporting electrolyte of citrate 0.25 mol L ${ }^{-1}$, EDTA $0.050 \mathrm{~mol} \mathrm{~L}^{-1}$ and $\mathrm{KNO}_{3} 0.50 \mathrm{~mol} \mathrm{~L}^{-1}$, pH 5.00. In this media iron (III) showed a polarographic peak in $-0.08 \mathrm{~V}$ and the copper (II) in $-0.34 \mathrm{~V}$, both vs. $\mathrm{Ag} / \mathrm{AgCl}$ (saturated $\mathrm{KCl}$ ). Thus, a analytical method was developed and applied to determine iron (III) in brass alloy, a matrix were copper is in large excess over iron. The results obtained showed no interference of copper in the iron determination. The value of $0.21 \%$ of iron in the sample alloy composition was obtained and the method was validated by atomic absortion and recovery test, and the results exhibited a good agreement with the proposed method.
\end{abstract}

Keywords: iron (III) determination; differential pulse polarography; brass analysis.

\section{INTRODUÇÃO}

Os métodos polarográficos/voltamétricos encontram inúmeras aplicações nos campos de análise inorgânica e orgânica, envolvem a utilização de diversos materiais ${ }^{1,2}$ e talvez sejam os métodos mais versáteis de análise de traços.

$\mathrm{Na}$ análise inorgânica, entre os metais estudados por esses métodos encontra-se o ferro, devido à sua grande importância e à necessidade de se dispor de métodos analíticos suficientemente sensíveis para a sua determinação em diversos tipos de matrizes $^{3}$. Por isso, vários métodos eletroanalíticos tem sido desenvolvidos para a determinação de ferro, tanto polarográfi$\cos ^{4-8}$ quanto voltametricos ${ }^{9-14}$, entre outros, visando não apenas a busca de uma maior sensibilidade mas também a eliminação de interferências que possam ocorrer nos eletrólitos de suporte utilizados ${ }^{6}$

Neste trabalho foi utilizada a técnica da polarografia de pulso diferencial para o estudo do ferro (III). Para isto foram examinados diversos aspectos das condições experimentais envolvendo o eletrólito de suporte, tendo em vista tanto os problemas de sensibilidade quanto os de interferência.

Entre as principais dificuldades encontradas nas determinações polarográficas de ferro em diversos eletrólitos de suporte, em relação aos problemas de interferentes, envolvem a sobreposição do potencial de meia onda, $\mathrm{E}_{1 / 2}$ do $\mathrm{Fe}(\mathrm{III})$ ou do seu potencial de pico, $\mathrm{E}_{\mathrm{p}}$, dependendo da técnica utilizada, com outras espécies, principalmente o $\mathrm{Cu}(\mathrm{II})^{15}$. Tal fato pode ser resolvido pela adição de complexantes ${ }^{6,16}$, que podem promover os deslocamentos dos potenciais de meia onda de modo a possibilitar a medição polarográfica sem a interferência. Deste modo, foram estudadas as condições experimentais envolvendo um eletrólito de suporte de forma que o cobre mesmo em grande excesso não produzirá interferências na determinação do ferro e vice-versa, devido à proximidade de seus potenciais de meiaonda ou de pico. A obtenção de condições experimentais onde a determinação quantitativa de $\mathrm{Fe}$ (III) ou $\mathrm{Cu}$ (II) possa ser feita sem estas interferências é um aspecto muito importante, uma vez que o $\mathrm{Cu}(\mathrm{II})$ ocorre em diversas matrizes conjuntamente com o $\mathrm{Fe}$ (III).

Outro aspecto importante considerado neste trabalho foi procurar desenvolver um método de análise no qual também possam ser reduzidas etapas no procedimento experimental envolvendo o tratamento prévio da amostra. Assim, tanto o $\mathrm{Fe}$ (III) quanto o $\mathrm{Cu}(\mathrm{II})$ poderão ser determinados de uma maneira mais simples, sem a necessidade de remoção prévia de um ou de outro na matriz a ser analisada .

Uma vez desevolvido o método, ele foi utilizado na determinação de ferro em latão, que se constitui em uma matriz em que o cobre está em grande excesso em relação ao ferro.

\section{PARTE EXPERIMENTAL}

Todos os reagentes utilizados foram de grau analítico, sem nenhuma purificação adicional. Todas as soluções foram preparadas e padronizadas de acordo com os procedimentos usuais encontrados na literatura salvo em situações especificadas.

A aparelhagem utilizada consistiu-se de um polarógrafo Radiometer Copenhagen modelo POL 150, acoplado ao "stand" Radiometer Copenhagen modelo MDE 150, em uma célula de três eletrodos, sendo o eletrodo de mercúrio o eletrodo de trabalho, o eletrodo de $\mathrm{Ag} / \mathrm{AgCl}, \mathrm{KCl}$ sat. o eletrodo de referência e um fio de platina o eletrodo auxiliar. O polarógrafo é conectado à um microcomputador $\mathrm{PC}$ com sistema operacional Windows $95^{\circledR}$, onde utilizou-se o programa Trace Master X9737-2.03, da Radiometer, para a realização das medições experimentais.

As medidas de $\mathrm{pH}$ foram realizadas em um $\mathrm{pH}$-metro digital Radelkis OP-271 com um eletrodo de vidro combinado também da marca Radelkis. O eletrodo foi calibrado com tampões NBS de pH 4,01 e 6,86 respectivamente. $\mathrm{O}$ pH das soluções do eletrólito de suporte foi ajustado adicionando-se $\mathrm{NH}_{3}$ ou $\mathrm{HCl}$ com a ajuda de um conta gotas a essas soluções até atingir-se o valor desejado.

O eletrólito de suporte foi uma solução de ácido cítrico 0,25

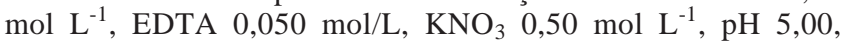
ajustado com amônia concentrada. A técnica polarográfica utilizada foi a polarografia de pulso diferencial, usando-se como eletrodo de trabalho o eletrodo de mercúrio no modo de gota estática, com tempo de gota de 1 segundo, amplitude de pulso de $-50 \mathrm{mV}$, duração de pulso de $40 \mathrm{~ms}$, velocidade de varredura de $5 \mathrm{mV} / \mathrm{s}$., corrente mínima de $10 \mathrm{nA}$ e máxima de 1 $\mathrm{mA}$. Os polarogramas foram obtidos no intervalo de potencial 
de $50 \mathrm{mV}$ a $-150 \mathrm{mV}$ para o $\mathrm{Fe}(\mathrm{III})$ e de $50 \mathrm{mV}$ a $-500 \mathrm{mV}$ quando se pretendia verificar a presença de $\mathrm{Fe}(\mathrm{III})$ e de $\mathrm{Cu}(\mathrm{II})$.

Para a determinação do ferro no latão abriu-se inicialmente uma amostra de 0,5825 g de latão usualmente encontrado no comércio de acordo com procedimento da literatura ${ }^{15}$, e após a sua dissolução, a amostra foi transferida para um balão volumétrico de $100,0 \mathrm{~mL}$ e díluída até a marca com água desionizada, sendo então estocada em um frasco previamente limpo e seco.

As medições experimentais foram feitas adicionando-se $10,00 \mathrm{~mL}$ do eletrólito de suporte na célula polarográfica, desaerando-se por 5 minutos com nitrogênio gasoso para a remoção do oxigênio atmosférico, registrando-se então o polarograma do eletrólito de suporte. A seguir, com a ajuda de uma micropipeta, adicionava-se à célula polarográfica um volume adequado da solução da amostra desaerando-se por mais 60 segundos e registrando-se o polarograma. Em seguida, adicionava-se um volume adequado de solução 0,01083 mol L ${ }^{-1}$ de $\mathrm{Fe}(\mathrm{III})$, desaerando-se por mais 60 segundos e registrando-se novamente o polarograma. Mais duas novas adições de solução padrão de $\mathrm{Fe}(\mathrm{III}) \quad 0,01083 \mathrm{~mol} \mathrm{~L}^{-1}$ procedendo da mesma forma que na adição anterior eram feitas para determinar-se o ferro na amostra pelo método da adição de padrão ${ }^{17}$. Todo o material de vidro utilizado foi limpo com solução de $\mathrm{HNO}_{3} 6 \mathrm{~mol} \mathrm{~L}{ }^{-1}$ e após, lavados várias vezes com água desionizada.

\section{RESULTADOS E DISCUSSÃO}

Os estudos iniciais foram feitos no sentido de se buscar um eletrólito de suporte adequado e encontrar as melhores condições para a determinação de $\mathrm{Fe}(\mathrm{III})$ sem as sobreposições de seu pico polarográfico com o do $\mathrm{Cu}(\mathrm{II})$.

A questão de interferências na determinação de Fe(III) em diversos eletrólitos de suporte e o uso de complexantes para procurar resolver este problema tem aparecido na literatura. Entre esses estudos encontra-se o de Souchay e Faucherre ${ }^{16}$ que usaram um eletrólito de suporte contendo EDTA 0,1 mol $\mathrm{L}^{-1}$, acetato de sódio $2 \mathrm{~mol} \mathrm{~L}^{-1}$, com o $\mathrm{pH}$ ao redor de 6 e 7 , para determinar pequenas quantidades de ferro na presença de grandes quantidades de cobre, como em latão. Bond e PFund ${ }^{6}$ usaram um eletrólito de suporte à base de citrato e EDTA como complexante para evitar interferências na determinação de ferro em banhos à base de zinco. Visalakshi e colaboradores ${ }^{5}$ empregaram um eletrólito de suporte de ácido acético/acetato $0,1 \mathrm{~mol} \mathrm{~L}^{-1}, \mathrm{KNO}_{3} 0,1 \mathrm{~mol} \mathrm{~L}^{-1}$, EDTA $0,1 \mathrm{~mol} \mathrm{~L}^{-1}$, pH 4,0, onde os complexos de $\mathrm{Fe}$ (II) e $\mathrm{Fe}$ (III) foram estudados. O $\mathrm{KNO}_{3}$ foi usado para ajustar a força iônica.

A partir desses estudos foram feitas diversas investigações com eletrólitos à base de citrato ou acetato, EDTA e $\mathrm{KNO}_{3}$. Os resultados obtidos indicaram o eletrólito de suporte constituído de $0,25 \mathrm{~mol} \mathrm{~L}^{-1}$ de citrato, 0,50 $\mathrm{mol} \mathrm{L}^{-1}$ de $\mathrm{KNO}_{3}, 0,050$ mol L $\mathrm{L}^{-1}$ de EDTA, pH 5,00, como aquele que apresentou as melhores condições para a determinação de $\mathrm{Fe}(\mathrm{III})$ e de $\mathrm{Cu}$ (II) na presença um do outro.

A Figura 1 mostra os polarogramas de pulso diferencial devido ao $\mathrm{Fe}(\mathrm{III})$ e ao $\mathrm{Cu}(\mathrm{II})$. Na Figura 1a, o eletrólito de suporte usado foi o citrato $0,25 \mathrm{~mol} \mathrm{~L}^{-1}, \mathrm{KNO}_{3} 0,50 \mathrm{~mol} \mathrm{~L}^{-1}$, $\mathrm{pH} 5,00$. Em 10,00 mL deste eletrólito foram adicionados $200,0 \mu \mathrm{L}$ de $\mathrm{Fe}(\mathrm{III}) 0,01083 \mathrm{~mol} \mathrm{~L}^{-1}$ e $100,0 \mu \mathrm{L}$ de $\mathrm{Cu}(\mathrm{II})$ $0,00973 \mathrm{~mol} \mathrm{~L}^{-1}$, sendo que apenas um pico polarográfico foi registrado em $-0,10 \mathrm{~V}$. Na Figura $1 \mathrm{~b}$ as mesmas quantidades de cobre e ferro foram adicionadas no eletrólito de suporte citrato $0,25 \mathrm{~mol} \mathrm{~L}^{-1}, \mathrm{KNO}_{3} 0,50 \mathrm{~mol} \mathrm{~L}{ }^{-1}$, EDTA $0,050 \mathrm{~mol} \mathrm{~L}^{-1}$, $\mathrm{pH} 5,00$, sendo que agora dois picos polarográficos foram registrados, um em $-0,08 \mathrm{~V}$, devido ao $\mathrm{Fe}(\mathrm{III})$, e o outro em $-0,34 \mathrm{~V}$, devido ao $\mathrm{Cu}(\mathrm{II})$. Esses dados mostram que a ação complexante do EDTA causa a separação dos picos polarográficos devido aos complexos $\mathrm{Fe}$ (III)-EDTA e $\mathrm{Cu}$ (II)-EDTA. Essa ação complexante do EDTA que produz o deslocamento dos potenciais de pico pode ser explicada em função dos complexos das diversas espécies desse metais envolvidas nos processos eletródicos. Assim, no caso do ferro, necessita-se considerar as formas $\mathrm{Fe}(\mathrm{III})$ e $\mathrm{Fe}(\mathrm{II})$. O EDTA forma complexos com essas duas espécies, e o valor do $\Delta \mathrm{E}_{1 / 2}$ dependerá principalmente da razão das constantes de formação dos complexos de $\mathrm{Fe}(\mathrm{III})$ e $\mathrm{Fe}$ (II) no meio utilizado. No caso do cobre, a forma em solução é somente $\mathrm{Cu}(\mathrm{II})$, que se reduz a $\mathrm{Cu}^{0}$ no eletrodo de mercúrio, nesse caso o deslocamento do seu potencial de pico no sentido catódico deverá ser maior que no caso do $\mathrm{Fe}(\mathrm{III})$, que praticamente não se desloca, o que é confirmado pelos dados acima mencionados.

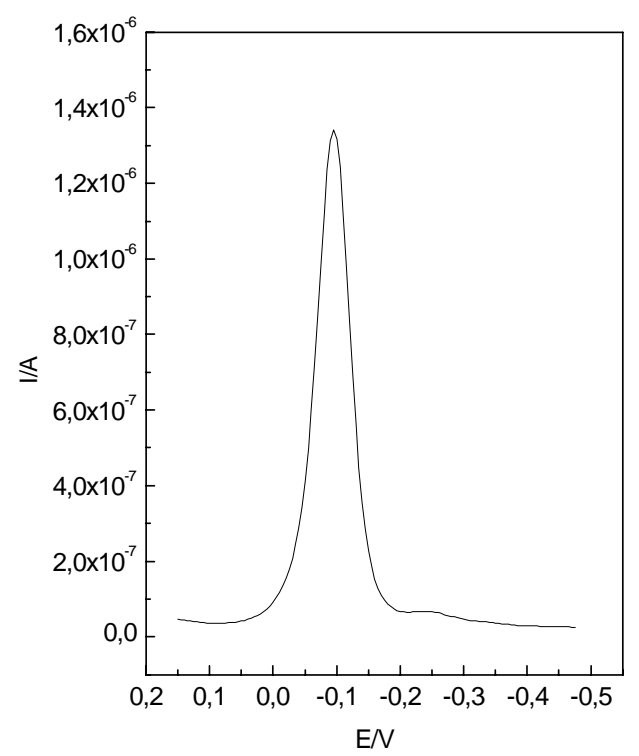

b

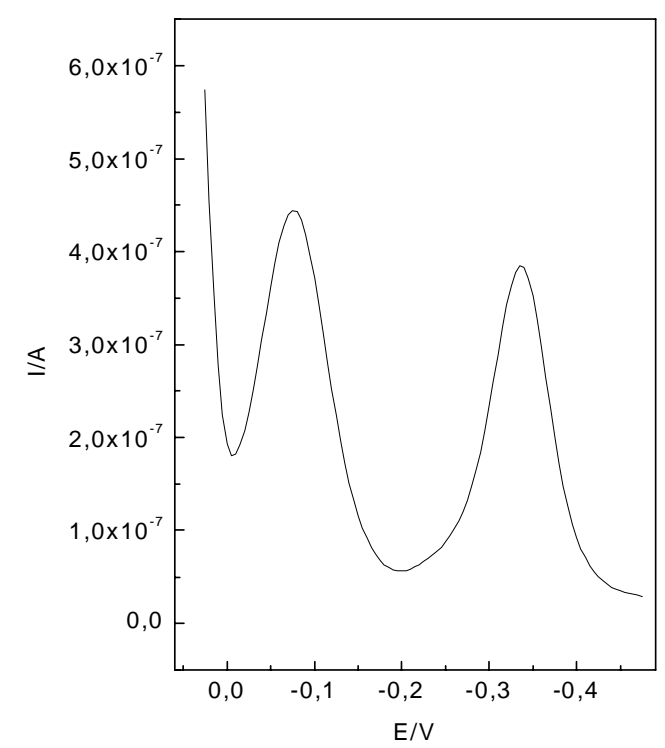

Figura 1. Polarogramas referentes à ação complexante do EDTA sobre a separação dos potenciais de pico ( $\left.E_{p}\right)$ de $F e(I I I)$ e Cu(II). (a), Eletrólito de suporte: Citrato 0,25 mol L $L^{-1} \mathrm{KNO}_{3} 0,50 \mathrm{~mol} \mathrm{~L} \mathrm{~L}^{-1}, \mathrm{pH}$ 5,00. (b), Eletrólito de suporte: Citrato 0,25 mol L $\mathrm{L}^{-1}, \mathrm{KNO}_{3}$ $0,50 \mathrm{~mol} \mathrm{~L}{ }^{-1}$, EDTA 0,050 mol L $L^{-1}$ pH 5,00. [Fe(III)] $=2,10.10^{-4} \mathrm{~mol} \mathrm{~L} L^{-1} ;[\mathrm{Cu}(\mathrm{II})]=9,44.10^{-5} \mathrm{~mol} \mathrm{~L}^{-1}$, em (a) e (b). 
$\mathrm{O}$ efeito do $\mathrm{pH}$ sobre os potenciais de pico de $\mathrm{Fe}(\mathrm{III})$ e $\mathrm{Cu}$ (II) foi estudado para a verificação da região em que a melhor separação entre eles pudesse ser obtida. $\mathrm{O}$ pH do eletrólito de suporte foi variado no intervalo de 3,80 a 5,40, a cada 0,20 unidades, e em cada $\mathrm{pH}$ foram registrados os polarogramas relativos ao $\mathrm{Fe}(\mathrm{III})$ e ao $\mathrm{Cu}(\mathrm{II})$, determinando-se os valores de seus potenciais de pico. Na Tabela 1 são apresentados os valores desses pontencias em função do $\mathrm{pH}$, bem como os valores referentes à diferença $\left(\Delta \mathrm{E}_{\mathrm{p}}\right)$ entre esses potenciais.

Tabela 1. Variação dos valores de Ep do $\mathrm{Fe}(\mathrm{III})$ e de Ep do $\mathrm{Cu}(\mathrm{II})$ em função do $\mathrm{pH}$. Valores de Ep obtidos para o eletrólito de suporte citrato /ácido cítrico $0,25 \mathrm{~mol} \mathrm{~L}^{-1} ; \mathrm{KNO}_{3} 0,50 \mathrm{~mol} \mathrm{~L}^{-1} \mathrm{e}$ EDTA $0,050 \mathrm{~mol} \mathrm{~L}^{-1}$ (pH ajustado com amônia concentrada).

\begin{tabular}{cccc}
\hline $\mathrm{pH}$ & $\mathrm{Ep} \mathrm{Fe}(\mathrm{III})(\mathrm{mV})$ & $\mathrm{Ep} \mathrm{Cu}(\mathrm{II})(\mathrm{mV})$ & $\Delta \mathrm{Ep}(\mathrm{mV})$ \\
\hline 3,8 & -50 & -245 & 195 \\
4,0 & -55 & -260 & 205 \\
4,2 & -55 & -275 & 215 \\
4,4 & -55 & -285 & 230 \\
4,5 & -55 & -290 & 235 \\
4,6 & -55 & -300 & 245 \\
4,7 & -55 & -300 & 245 \\
4,8 & -55 & -305 & 250 \\
4,9 & -55 & -310 & 255 \\
5,0 & -55 & -310 & 255 \\
5,1 & -55 & -310 & 255 \\
5,2 & -55 & -320 & 265 \\
5,3 & -50 & -325 & 275 \\
5,4 & -55 & -325 & 280 \\
\hline
\end{tabular}

A Figura 2 mostra o gráfico de $\Delta \mathrm{E}_{\mathrm{p}}$ vs. $\mathrm{pH}$. A melhor região de $\mathrm{pH}$ verificada para o eletrólito de suporte foi entre $\mathrm{pH} 4,90$ e 5,10 . Nesta região o $\Delta \mathrm{E}_{\mathrm{p}}$ tende a ficar constante e a separação dos picos do $\mathrm{Fe}(\mathrm{III})$ e $\mathrm{Cu}(\mathrm{II})$ torna-se bem definida. Em valores de $\mathrm{pH}$ menor que 4,90 foi verificado que os picos tendem a aproximar-se e a ocorrer sobreposições entre eles, e em pHs maiores que 5,10, o pico do $\mathrm{Fe}$ (III) tende a diminuir e a ser sobreposto pela corrente anódica devido a oxidação do mercúrio. A Figura 3 mostra polarogramas de $\mathrm{Fe}(\mathrm{III})$ e $\mathrm{Cu}$ (II) em valores de $\mathrm{pH} 3,80,5,00$ e 5,40, ilustrando essas três regiões de $\mathrm{pH}$ estudadas.

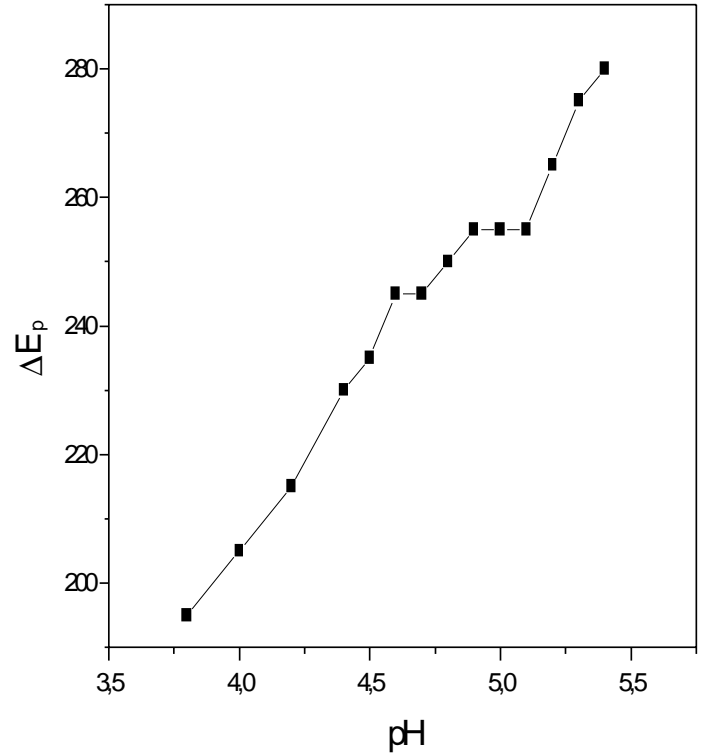

Figura 2. Diferença de potenciais de pico $\left(\Delta E_{p}\right)$ de $F e(I I I)$ e $\mathrm{Cu}(I I)$ em função do $\mathrm{pH} . \Delta E_{p}=\left(E_{p F e(I I I)}-E_{p C u(I I I)}\right)$, eletrólito de suporte, citrato $0,25 \mathrm{~mol} \mathrm{~L} L^{-1}, \mathrm{KNO}_{3} 0,50 \mathrm{~mol} \mathrm{~L} \mathrm{~L}^{-1}$, EDTA $0,050 \mathrm{~mol} L^{-1} \cdot[\mathrm{Fe}(\mathrm{III})]=2,10.10^{-4} \mathrm{~mol} L^{-1} ;[\mathrm{Cu}(\mathrm{II})]=$ $9,53 \cdot 10^{-5} \mathrm{~mol} L^{-1}$.

Tendo-se definidas estas condições experimentais, foi construída uma curva de calibração corrente de pico vs. concentração de $\mathrm{Fe}(\mathrm{III})$. Essa curva mostrou um comportamento linear na região de concentração estudada, entre $2,5 \times 10^{-6}$ e $2,4 \times 10^{-5} \mathrm{~mol} \mathrm{~L}^{-1}$, apresentando um coeficiente de correlação de 0,999 . O limite de deteção calculado de acordo com Miller e Miller ${ }^{18}$, considerandose 3 vezes o desvio padrão do branco, foi de $6,6 \times 10^{-6} \mathrm{~mol} \mathrm{~L}^{-1}$. Esse comportamento linear assegura que pode-se aplicar o método da adição de padrão nessa faixa de concentração.

Uma vez estabelecida as melhores condições de trabalho com o eletrólito de suporte, procedeu-se à determinação de ferro no latão. Inicialmente procurou-se determinar a melhor proporção matrizeletrólito a ser utilizada na célula polarográfica. Verificou-se que esta proporção foi a de uma solução ao redor de 0,5 a $0,6 \%$ (na faixa de 5 a $6 \mathrm{~g} \mathrm{~L}^{-1}$ ) de latão na célula polarográfica, onde o pico do Fe(III) obtido nos polarogramas apresentava-se bem definido.
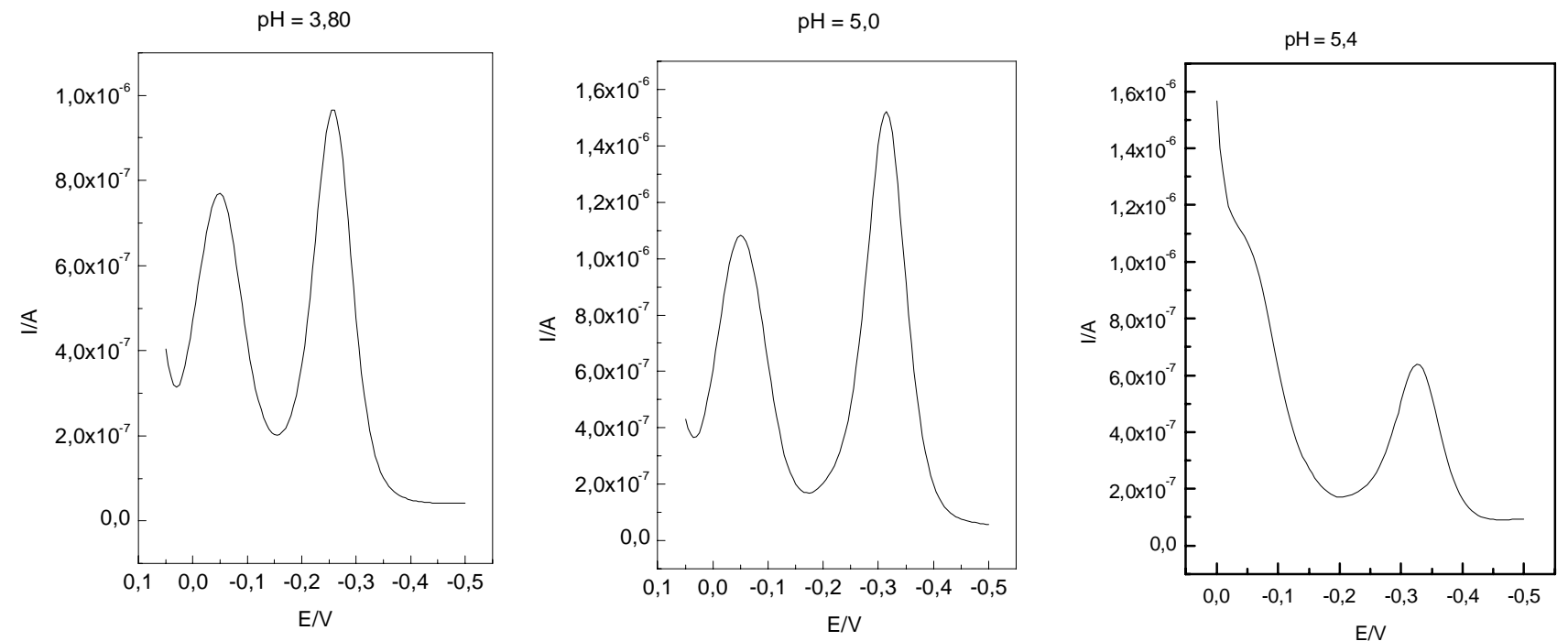

Figura 3. Polarogramas para o Fe(III) e Cu(II) relativos aos valores de pH 3,80, 5,00 e 5,40, respectivamente, no eletrólito de

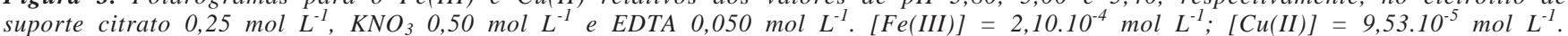


A determinação do ferro no latão foi então feita pelo método da adição de padrão, conforme mencionado na parte experimental. Explicando-se mais detalhadamente este método, procedeu-se para este fim registrando-se inicialmente o polarograma da amostra contida na célula polarográfica. Em seguida adicionou-se à amostra uma quantidade conhedida da solução padrão de Fe(III), registrando-se o polarograma da amostra mais o padrão. Seguindo-se com este procedimento, foram feitas mais duas adições de padrão, registrando-se o respectivo polarograma correspondente à cada adição. Para cada determinação, portanto, foram feitas três adições de padrão. A concentração do ferro foi então determinada via gráfico conforme pode ser visto na Figura 4. A curva referente a este gráfico é feita colocando-se no mesmo os valores de corrente de pico $\mathrm{I}_{\mathrm{p}}$ vs. concentração, conforme indicado a seguir. $\mathrm{O}$ valor de $\mathrm{I}_{\mathrm{p}}$ referente à amostra é colocado no eixo y correspondente ao valor zero do eixo $\mathrm{x}$. Na curva, à direita do valor de $\mathrm{x}$ igual a zero, colocam-se os pontos correspondentes aos valores de $\mathrm{I}_{\mathrm{p}}$ (eixo y) vs. concentração dos padrões (eixo x). À esquerda do valor zero do eixo $\mathrm{x}$, a escala de concentração neste eixo $\mathrm{x}$ é a mesma que a da direita. Assim, ao traçar-se a reta referente aos pontos experimentais, no lugar onde ela interceptar o eixo x corresponderá à concentração de $\mathrm{Fe}$ (III) referente à amostra de latão na célula polarográfica (neste ponto $\mathrm{I}_{\mathrm{p}}$ será igual a zero). A partir daí calcula-se a concentração de ferro na solução original da matriz chegando-se então à porcentagem do ferro no latão.

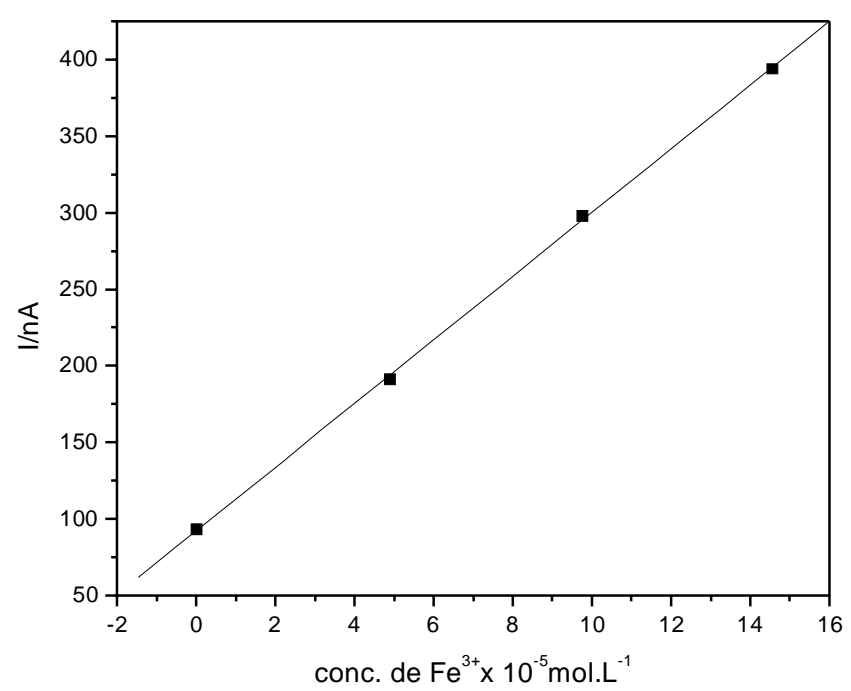

Figura 4. Determinação de ferro em latão pelo método da adição de padrão. Gráfico corrente de pico $\left(I_{p}\right)$ vs. concentração de Fe(III). Dados experimentais referentes à tabela 2.

Por este procedimento foi analisada uma solução de uma amostra 5,531 $\mathrm{g} \mathrm{L}^{-1}$ de latão. Os resultados são apresentados na tabela 2 . A determinação foi repetida 5 vezes e um valor de
$0,21 \% \pm 0,01 \%$ de ferro foi encontrado para a composição da liga, com $n=5$ e intervalo de confiança de $95 \%$. Na Figura 5 são mostrados polarogramas de uma destas determinações.

A validação do método foi realizada pelo teste de recuperação e pela determinação do ferro por absorção atômica ${ }^{19}$. $\mathrm{O}$ teste de recuperação foi feito adicionando-se uma quantidade conhecida de $\mathrm{Fe}(\mathrm{III})$ a partir de uma solução padrão à amostra de latão previamente analisada. Em seguida, a determinação do ferro total foi feita pelo método da adição de padrão. Da concentração assim obtida foi subtraída a concentração da amostra e verificada a recuperação da concentração de $\mathrm{Fe}$ (III) adicionada em termos de porcentagem. Os resultados obtidos são mostrados na Tabela 3 e como pode ser visto os valores das porcentagens de recuperação são inteiramente satisfatórios.

A determinação do ferro no latão por absorção atômica apresentou um resultado de $0,21 \% \pm 0,02 \%$ para o ferro na composição do latão, o qual concorda inteiramente com o valor de $0,21 \% \pm 0,01 \%$ encontrado polarograficamente, o que juntamente com o teste de recuperação, valida o método proposto.

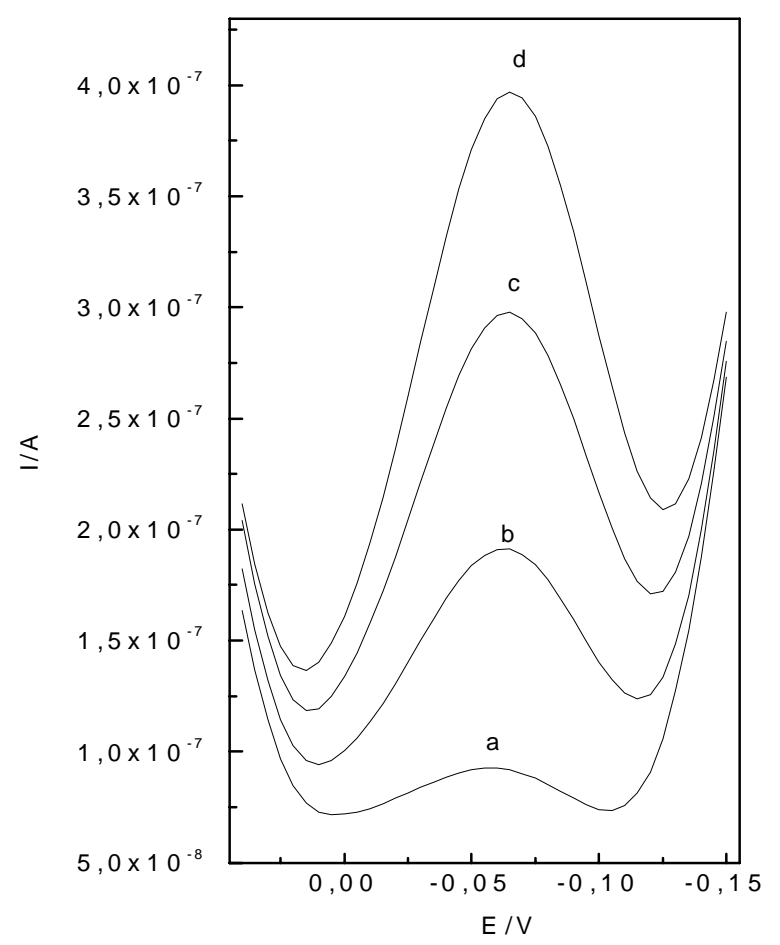

Figura 5. Polarogramas referentes à determinação de ferro em latão. Dados relativos à tabela 2 (Det. 1). (a) amostra; (b) amostra + 50,0 $\mu \mathrm{L}$ de $\mathrm{Fe}(\mathrm{III})$ 0,01083 mol $L^{-1} ;$ (c) amostra $+2 \times 50,0 \mu \mathrm{L} d e$ $\mathrm{Fe}(\mathrm{III})$ 0,01083 mol L $\mathrm{L}^{-1}$ (d) amostra $+3 \times 50,0 \mu \mathrm{L}$ de $\mathrm{Fe}(\mathrm{III})$ $0,01083 \mathrm{~mol} \mathrm{~L}^{-1}$.

Tabela 2. Determinação da \% de Fe(III) em latão pelo método de adição de padrão. Valores de Ip ( corrente de pico) vs. concentração de $\mathrm{Fe}(\mathrm{III})$ padrão.

\begin{tabular}{|c|c|c|c|c|c|}
\hline & \multicolumn{5}{|c|}{ Determinação de $\mathrm{Fe}(\mathrm{III})$ pelo método de adição de padrão (Det.) } \\
\hline & Det.1 & Det.2 & Det.3 & Det.4 & Det. 5 \\
\hline$[\mathrm{Fe}(\mathrm{III})] \times 10^{-5} \mathrm{~mol} . \mathrm{L}^{-1}$ & $\operatorname{Ip}(\mathrm{nA})$ & $\operatorname{Ip}(\mathrm{nA})$ & $\operatorname{Ip}(n A)$ & $\operatorname{Ip}(\mathrm{nA})$ & $\operatorname{Ip}(\mathrm{nA})$ \\
\hline 0 & 93 & 98 & 101 & 93 & 84 \\
\hline 4,90 & 191 & 196 & 205 & 227 & 184 \\
\hline 9,76 & 298 & 297 & 280 & 364 & 288 \\
\hline 14,6 & 394 & 400 & 371 & 483 & 388 \\
\hline $\mathrm{R}$ & 0,999 & 0,999 & 0,999 & 0,999 & 0,999 \\
\hline$\%$ de $\mathrm{Fe}(\mathrm{III})$ na liga & 0,21 & 0,22 & 0,21 & 0,21 & 0,21 \\
\hline
\end{tabular}


Tabela 3. Teste de Recuperação para a determinação polarográfica de ferro em latão. Valores relativos a concentração de $\mathrm{Fe}(\mathrm{III})$ total na cela polarográfica.

\begin{tabular}{ccc}
\hline$\mu \mathrm{gFe}(\mathrm{III}) \cdot \mathrm{L}^{-1}$ (esperado) & $\mu \mathrm{gFe}(\mathrm{III}) \cdot \mathrm{L}^{-1}$ (obtido) & $\%$ de Recuperação \\
\hline 63,60 & 62,93 & 97 \\
84,60 & 83,75 & 98 \\
105,60 & 106,66 & 102 \\
126,60 & 129,13 & 103 \\
\hline
\end{tabular}

\section{CONCLUSÃO}

$\mathrm{O}$ estudo do comportamento polarográfico do $\mathrm{Fe}(\mathrm{III})$ e do $\mathrm{Cu}(\mathrm{II})$ no eletrólito de suporte citrato $0,25 \mathrm{~mol} \mathrm{~L}^{-1}, \mathrm{KNO}_{3} 0,50$

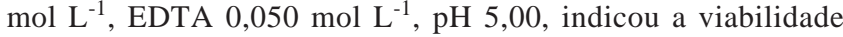
da determinação desses metais, em suas formas complexadas com EDTA, na presença um do outro, uma vez que a sobreposição dos picos polarográficos dessas espécies deixa de ocorrer nessas condições.

Baseado nesses resultados foi feita uma aplicação do método desenvolvido na determinação de ferro em uma amostra real. A amostra escolhida foi o latão, uma liga metálica à base de cobre, o qual está presente na liga em grande excesso em relação ao ferro, sendo portanto um material ideal para testar o método. Os bons resultados obtidos mostraram a validade do método desenvolvido.

Em razão do presente trabalho ter sido bem sucedido, estão sendo feitos diversos estudos buscando-se aplicar este método polarográfico na determinação de ferro em outras matrizes. Devido as propriedades apresentadas pelo eletrólito de suporte à base de citrato/EDTA, além de se procurar determinar ferro na presença de cobre, estão sendo realizados estudos sobre a determinaçõe de cobre na presença de ferro e também de ambos os metais simultaneamente.

\section{AGRADECIMENTOS}

Os autores agradecem à FAPESP e ao CNPq pela ajuda financeira e ao Prof. José S. Barone pelas análises de ferro usando-se absorção atômica. M. S. agradece ao CNPq pela bolsa recebida.

\section{REFERÊNCIAS}

1. Bond, A. M.; Modern Polarographic Methods in Analytical Chemistry, M. Dekker, New York; 1980.

2. Wang, J.; Stripping Analysis, VCH Publishers, Deerfield Beach; 1985.

3. Lu, J.; Wang, J.; Yarnitzky, C.; Electroanalysis 1995, 7, 79.

4. Hernández, L.; Zapardiel, A.; Pérez-Lópes, J. A.; Bermejo, E.; Anal. Chim. Acta 1987,198, 239.

5. Visalakshi, G.; Narasimhan, S. V.; Venkateswarlu, K. S.; Anal. Chim. Acta 1988, 212, 335.

6. Bond, A. M.; Pfund, B. V.; Anal. Chim. Acta 1993, 277, 145.

7. Bharatibai, J. B.; Padma, D. K.; Rajagopalan, S. R.; Fresenius J. Anal. Chem. 1994, 349, 477.

8. Wang, S.; Du, L.; Bin Li, A. Z.; Anal. Lett. 1997, 30, 2099.

9. Cox, J. A.; Majda, M.; Anal. Chim. Acta 1980, 118, 271.

10. Van Den Berg, C. M. G.; Huang, Z. Q.; J. Electroanal. Chem. 1984, 177, 269.

11. Wang, J.; Mahmoud, J.; Fresenius Z. Anal. Chem. 1987, $327,789$.

12. Hua, C.; Jagner, D.; Renman, L.; Talanta 1988, 35, 597.

13. Gao, Z.; Kok, S. S.; Talanta 1996, 43, 727.

14. El-Maali, N. A.; Temerk, Y. M.; El-Aziz, M. S. A.; Anal. Chim. Acta 1997, 353, 313.

15. Kolthoff, I. M.; Lingane, J. J.; Polarography, vol. II, Interscience Publishers, $2^{\mathrm{a}}$ ed., 1965, p 499 e p 583.

16. Souchay, P.; Faucherre, J.; Anal. Chim. Acta 1949, 3, 259.

17. Riley, T.; Watson, A.; Polarography and other Voltammetric Methods, John Wiley \& Sons, Chichester, 1987, p. 220, 221.

18. Miller, J. C.; Miller, J. N.; Statistics for Analytical Chemistry, Ellis Horwood, Chichester, $3^{\mathrm{a}}$ ed., 1990, p. 115-117.

19. Christian, G. D.; Analytical Chemistry, John Willey \& Sons, New York, 5a ed., 1994, p. 10, 11. 